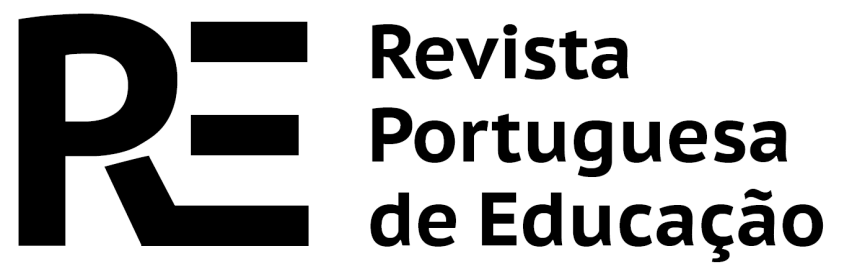

\title{
Adaptação cultural de um instrumento para avaliar as emoções do professor (TEQ)
}

RESUMO

O estudo das emoções dos professores tem vindo a ganhar destaque, sendo atualmente reconhecido como uma dimensão essencial do trabalho docente. Contudo, apesar de ter vindo a ganhar relevância na área educacional, verifica-se uma lacuna de instrumentos quantitativos válidos para mensurar as emoções discretas do professor. Este estudo teve como objetivo traduzir para português e adaptar o Teacher Emotion Questionnaire (TEQ) para a cultura brasileira. Foram envolvidos, 51 participantes, dos quais quatro tradutores, sete especialistas, 40 professores, e ainda as pesquisadoras e autora principal do TEQ original. $O$ presente estudo metodológico de adaptação cultural contou com as seguintes etapas: (1) traduções; (2) síntese das versões traduzidas; (3) comitê de especialistas; (4) avaliação pelo público-alvo; (5) tradução reversa; (6) estudo-piloto. As etapas tradução e síntese foram realizadas adequadamente, tendo sido introduzidas algumas alterações após a apreciação pelos especialistas e público-alvo. $O$ estudo piloto mostrou que o instrumento adaptado é de fácil compreensão e preenchimento. Por fim, após alguns ajustes, a adaptação cultural foi aprovada pela autora principal do TEQ original. 0 presente estudo realizou o primeiro passo para disponibilizar um instrumento confiável e válido sobre as emoções dos professores. 0 próximo passo será a exploração das propriedades psicométricas do TEQ Versão brasileira.

Palavras-chave: Emoções do professor; Adaptação cultural; Teacher Emotion Questionnaire

\section{INTRODUÇÃO}

O estudo das emoções dos professores foi, durante muito tempo, uma área de estudo negligenciada (Slišković et al., 2019). Contudo, esta tem vindo a ganhar destaque (Chen, 2016; Taxer \& Frenzel, 2015), sendo atualmente reconhecido que as emoções do professor são uma componente essencial do

Camila Marta de Almeida ${ }^{i}$ Universidade de Lisboa, Portugal

Sofia Freire ${ }^{i \mathbf{i}}$

Universidade de Lisboa, Portugal 
trabalho docente (Hargreaves, 2000; Schutz et al., 2006; Zembylas \& Schutz, 2009; Sutton \& Wheatley, 2003; Taxer \& Frenzel, 2015). Hargreaves (1998) entende mesmo, o ensino como uma prática emocional, e Zembylas (2007) refere que o conhecimento emocional sobre o ensino e a aprendizagem é uma componente essencial do conhecimento pessoal e prático do professor. Compreender as emoções, suas e dos outros no contexto educativo, e trabalhar as emoções, em particular a sua expressão, supressão e regulação em contextos específicos, são aspetos essenciais do trabalho do professor (Hargreaves, 1998; Taxer \& Frenzel, 2015; Zembylas, 2007).

Para além deste entendimento das emoções dos professores, vários estudos têm também vindo a revelar que as emoções experimentadas pelo professor em contexto escolar influenciam a sua cognição (e.g., atenção, memória e resolução de problemas), a sua motivação intrínseca para ensinar (Sutton \& Wheatley, 2003) e o próprio processo de ensino-aprendizagem (Chen, 2016; Zembylas \& Schutz, 2009), afetando a escolha de abordagens de ensino (Chen, 2019) e do próprio conteúdo a ensinar (Frenzel et al., 2009; Sheppard \& Levy, 2019). Em particular, a experiência de emoções negativas (raiva e ansiedade) no contexto de sala de aula está associada à utilização de estratégias de ensino mais inflexíveis. Em contrapartida, professores que experimentam emoções mais positivas (prazer) estão mais propensos a utilizar estratégias criativas no decorrer do processo de ensinoaprendizagem (Frenzel et al., 2009). No mesmo sentido, emoções positivas (alegria e amor) estão associadas a abordagens de ensino focadas no aluno e na interação aluno-professor. Já as emoções negativas (raiva, tristeza e medo), estão associadas a uma abordagem de ensino centrada na transmissão de conteúdo, que tem como foco o professor (Chen, 2019).

Para além da relação entre emoções e abordagens e estratégias de ensino do professor, diversos estudos têm, ainda, revelado que as experiências emocionais dos professores estão associados a outras dimensões do trabalho docente, tal como satisfação no trabalho e burnout docente (Burić et al., 2018; Frenzel et al., 2016; Hong et al., 2016; Slišković et al., 2019; Taxer \& Frenzel, 2015), envolvimento com o trabalho (Burić \& Macuka, 2017; Slišković et al., 2019) e autoeficácia docente (Burić et al., 2018; Taxer \& Frenzel, 2015).

Assim, o estudo das emoções dos professores é um importante campo de investigação, cujo conhecimento produzido poderá dar contributos significativos para a construção de ambientes educativos de melhor qualidade. Para além disso, poderá ser fundamental para apoiar professores em formação inicial e em serviço a compreender manifestações afetivas no contexto da sua prática educativa e a refletir sobre as suas implicações profissionais e pessoais (Chen, 2016). Contudo, para tal é fundamental desenvolver metodologias e instrumentos válidos que permitam identificar as emoções dos professores em contexto de sala de aula e de escola (Slišković et al., 2019; Xu, 2018).

No presente estudo, o conceito de emoção do professor é entendido segundo uma perspetiva componencial (Scherer, 2005). De acordo com esta perspetiva, a emoção é uma resposta à avaliação que o indivíduo faz de um estímulo interno ou externo que é importante para ele, avaliação essa que resulta de um conjunto de alterações relacionadas e simultâneas em diferentes sistemas, tais como cognitivo, neurofisiológico, motor e motivacional (Scherer, 2005). 
Os instrumentos existentes sobre a mensuração das emoções dos professores são, geralmente, de autorrelato, solicitando aos professores uma descrição/avaliação de emoções associadas aos alunos e ao ensino (Burić et al., 2018; Chen; 2016; Frenzel et al., 2016) e a fatores contextuais, como exemplo, colegas de trabalho, políticas educacionais (Chen, 2016). Este tipo de instrumentos tem-se revelado bastante útil, pois não só permite aceder aos aspetos subjetivos e cognitivos das emoções, como também a componentes motivacionais e expressivos das emoções (Burić, et al., 2018).

Alguns instrumentos de autorrelato destacados na literatura utilizados para mensurar as emoções discretas dos professores são: Teacher Emotions Scales (TES) (Frenzel et al., 2009); Inventory Teacher Emotion (TEI) (Chen, 2016); Teacher Emotion Questionnaire (Burić et al.,2018). O TES desenvolvido por Frenzel et al. (2016) é um instrumento que avalia três emoções dos professores em relação ao processo de ensino (prazer, raiva e ansiedade). É composto por doze itens, quatro itens para cada uma das três emoções. Essa escala foi validada nos idiomas inglês e alemão, sendo que atua da mesma maneira nas duas versões, com consistência interna que varia de 0.70 e 0.92. O TEI (Chen, 2016) avalia as emoções dos professores em relação aos alunos, aprendizagem, e ensino, e a fatores contextuais (e.g., pais, colegas de trabalho, sociedade e políticas educacionais). É composto por vinte e seis itens, e após análise fatorial exploratória e confirmatória, foram identificadas cinco emoções, duas das quais positivas (alegria e amor) e três das quais negativas (tristeza, raiva e medo). Essa escala mostrou-se confiável e válida na medição das emoções dos professores na China e Hong Kong, com uma consistência interna de 0.84 (Chen, 2019). Já o TEQ, idealizado por Burić et al. (2018), é um instrumento de autorrelato multidimensional sobre as emoções que os professores experimentam em relação ao ensino e na interação com os alunos, que avalia seis emoções (alegria, amor, orgulho, raiva, fadiga/exaustão e desesperança). A avaliação da consistência interna variou de 0.81 a 0.90 .

Embora os instrumentos apresentados sejam válidos e confiáveis, o TEQ parece ser o mais adequado por abranger seis emoções discretas do professor (alegria, orgulho, amor, raiva, exaustão/fadiga e desesperança) em relação ao ensino e à sala de aula. Pelo contrário, o TES abrange apenas três emoções do professor (prazer, raiva e ansiedade) e o TEI cinco emoções (alegria, amor, tristeza, raiva e medo). Nas sugestões de futuras pesquisas, os autores da escala TES ressaltam a necessidade de estudos posteriores abrangerem mais emoções (Frenzel et al., 2016), salientando, em particular, a emoção e o orgulho. Hong et al. (2016) sugerem também a inclusão de outras emoções, tais como a esperança, desesperança, culpa e vergonha. Afinal, a vida emocional do professor é composta por diversas emoções discretas (Taxer \& Frenzel, 2015). O TEQ se apresenta, assim, como um instrumento que dá resposta a estas lacunas, no que se refere a abranger um maior número de emoções discretas que a literatura vem apontando como significativas no estudo sobre as emoções do professor (e.g., orgulho e desesperança).

Muito embora o estudo das emoções dos professores tenha vindo a ganhar relevância na área educacional, no Brasil verifica-se uma lacuna de instrumentos quantitativos válidos para mensurar as emoções discretas do professor. Ao realizar um levantamento sistemático nas bases de dados Scielo, Periódicos Capes e Banco de teses Capes - com utilização dos 
descritores: emoção e professor, sentimento e professor, afeto e professor, afetividade e professor - não foi possível encontrar uma escala de autorrelato, validada, no idioma português, sobre emoções dos professores em relação ao contexto de sala de aula e ao ensino. Assim, o presente estudo teve como objetivo traduzir o TEQ para a língua portuguesa e adaptá-lo para a cultura brasileira.

Tendo em conta que a expressão, supressão e regulação de emoções em contextos específicos depende de fatores pessoais, do ambiente educativo do qual o professor faz parte e da maneira como a sociedade perceciona o papel da educação (Freire et al., 2014), mas também de aspetos culturais e políticos (Fried, Mansfield \& Dobozy, 2015; Hargreaves, 2000; Shutz et al., 2006), é fundamental proceder à adaptação cultural de instrumentos, já que essa adaptação permite comparar populações de diferentes culturas (Borsa et al., 2012). A adaptação cultural é um processo metodológico complexo e que envolve diversas etapas (Borsa et al., 2012) com o intuito de obter um instrumento válido e confiável (Beaton et al., 2000). Espera-se que o TEQ - Versão brasileira possa contribuir para a diminuição da lacuna de instrumentos para o estudo das emoções dos professores no Brasil, sendo utilizado para mensurar as emoções dos professores em relação ao processo de ensino e na interação com os alunos.

\section{METODOLOGIA}

\subsection{APRESENTAÇÃO DO INSTRUMENTO: TEACHER EMOTION QUESTIONNAIRE (TEQ)}

O TEQ, idealizado por Burić et al., (2018), na Croácia, é um instrumento de autorrelato multidimensional sobre as emoções que os professores experimentam em relação ao ensino e na interação com os alunos, que avalia seis emoções: alegria, amor, orgulho, raiva, fadiga/exaustão e desesperança. O TEQ surgiu a partir de um estudo de métodos mistos, que abrangeu cinco estudos, desde o estudo de abordagem qualitativa até a avaliação das propriedades psicométricas, com diferentes amostras de professores. No primeiro estudo, qualitativo, foram explorados as fontes e estrutura das emoções dos professores para a criação dos itens do TEQ. 0 segundo estudo teve o intuito de explorar a estrutura fatorial dos itens do TEQ. Os resultados das análises exploratórias apontaram para 34 itens. Já no terceiro estudo foi testado novamente a estrutura com a análise fatorial confirmatória e verificado as propriedades psicométricas. Após a análise dos resultados permanecerem 29 itens. No quarto estudo, procurou-se avaliar a validade de critério. Verificou-se relações significativas de características demográficas, satisfação no trabalho, trabalho emocional, exaustão emocional e bem-estar. No último estudo, o objetivo foi o aprimoramento do TEQ por meio de nova avaliação psicométrica, aplicação em amostras de professores de diferentes fases do ensino, inclusão da escala sobre a emoção amor com seis itens (com novas análises fatoriais) e, por fim, verificou-se a validade convergente do TEQ com as seguintes variáveis: autoeficácia, valor do trabalho docente, afeto no trabalho e intenção de abandonar a profissão. Os resultados mostraram correlações significativas no sentido teórico esperado. A análise da consistência interna de cada escala apresentou valores de Alfa de Cronbach 
entre 0.81 a 0.90. 0 TEQ é, assim, um instrumento confiável pelos parâmetros rígidos empregados em seu desenvolvimento (Burić et al., 2018).

O TEQ é composto por 35 itens subdivididos em seis escalas relacionadas as emoções: alegria (5 itens, ex. I am happy when I manage to motivate students to learn), amor (6 itens, ex. I love my students), orgulho (6 itens, ex. I feel like a winner when my students succeed), raiva (5 itens, ex. The reactions of some students frustrate me so much that I would rather just quit the job), fadiga/exaustão (7 itens, ex. At the end of my working day, I just want to rest) e desesperança (6 itens, ex. I feel I cannot do anything more to correct the behavior of some students). Na instrução, é solicitado ao professor avaliar o seu nível de concordância em relação a cada afirmação, sobre o que ele sente quando está ensinado e interagindo com os alunos, em uma escala Likert de 5 pontos (1=discordo totalmente, $2=$ discordo, $3=$ não concordo nem discordo, $4=$ concordo e $5=$ concordo totalmente) (Burić et al., 2018).

\subsection{PROCEDIMENTO DE ADAPTAÇÃO CULTURAL - TEQ}

Primeiramente, foi solicitado, via e-mail, à autora principal do instrumento TEQ autorização para a tradução e a adaptação do instrumento TEQ original, em inglês, para a língua portuguesa e para a realidade brasileira. A partir dessa autorização, iniciou-se o processo de tradução e adaptação cultural com base nos procedimentos propostos por Borsa et al. (2012) referente a adaptação de instrumentos psicólogos, bem com as recomendações de Beaton et al. (2000), World Health Organization (WHO, 2020) e International Test Commission (ITC, 2017).

A adaptação cultural contou com as seguintes etapas (figura 1): tradução do instrumento; síntese das versões traduzidas; avaliação pelo comitê de especialistas; avaliação pelo público-alvo; tradução reversa; realização do estudo-piloto. 
Figura 1

Fluxograma dos procedimentos utilizados para a adaptação cultural do TEQ

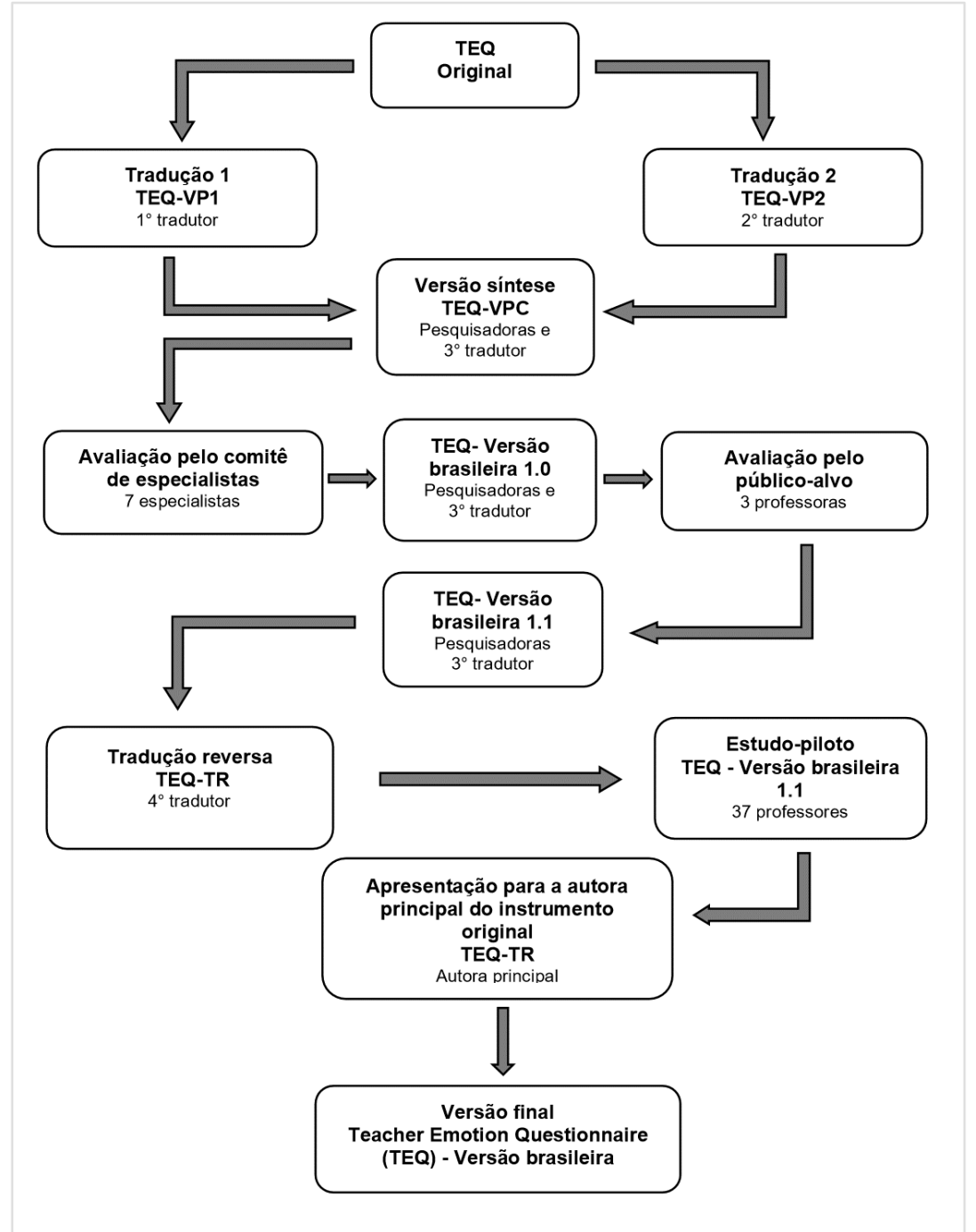

Nota. Adaptado de Borsa et al. (2012, p. 427).

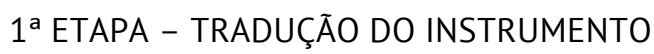

Participaram nesta fase dois tradutores profissionais, ambos bilíngues, tendo o português como língua materna e domínio da língua inglesa, e com ampla experiência na área de tradução. Um deles, bacharel em psicologia e mestrado na mesma área, com seis anos de experiência em tradução, e o segundo, bacharel em letras e com nove anos de experiência em tradução e revisão. Conforme recomendações de Beaton et. al (2000), um dos tradutores conhecia os objetivos e conceitos do estudo e tinha formação na área da psicologia. Já o segundo tradutor, denominado tradutor ingênuo, não tinha conhecimento sobre os objetivos do estudo e sua formação era na área de letras. Os tradutores realizaram a tradução, independente, do TEQ original para a língua portuguesa do Brasil. Assim, surgiram duas versões do TEQ traduzidas para o português: TEQ - Versão português 1 (TEQ-VP1) e TEQ Versão português 2 (TEQ-VP2).

\section{2. ${ }^{a}$ ETAPA - SÍNTESE DAS VERSÕES TRADUZIDAS}

Para a construção da versão síntese, participaram as duas pesquisadoras e um terceiro tradutor nativo na língua portuguesa e com domínio da língua inglesa. As TEQ-VP1 e TEQ-VP2 foram avaliadas pelas duas pesquisadoras e o 
terceiro tradutor com o objetivo de criar a versão síntese. A avaliação consistiu em analisar as duas traduções realizadas, na 1a etapa, na busca de uma adaptação para a população brasileira. Dessa maneira, após a realização de algumas reuniões, foi elaborado a Versão Português Consenso, denominada de TEQ-VPC.

\section{3. ${ }^{a}$ ETAPA - AVALIAÇÃO PELO COMITÊ DE ESPECIALISTAS}

A versão do instrumento, TEQ-VPC, foi submetida para nova avaliação por um comitê de especialistas, constituído por sete profissionais, com formação na área de psicologia $(n=4)$ e na área de pedagogia $(n=3)$, com domínio da língua inglesa, sendo seis mestres (em educação e psicologia). Os especialistas verificaram as equivalências semântica, idiomática, experiencial e conceitual da TEQ-VPC. Na equivalência semântica, verifica-se o significado das palavras (i.e., se estas possuem o mesmo significado que as palavras no item original) e aspetos gramaticais (por exemplo, a existência de possíveis erros na tradução). Na equivalência idiomática, é verificado se o termo (ou expressão) traduzido é equivalente ao termo original e se avalia a mesma emoção que na escala de origem. Na equivalência experiencial, é verificado se o item em si, independentemente da tradução, faz sentido no novo contexto cultural. A equivalência conceitual tem por objetivo verificar se o item (ou a expressão traduzida) avalia o mesmo aspeto cultural do item da escala original (Beaton et al., 2000; Borsa et al., 2012).

Foi enviado um instrumento, via e-mail, para os especialistas composto por uma carta de apresentação e pelo questionário TEQ-VPC. Os especialistas avaliaram as equivalências citadas, numa escala de três pontos (variando entre $-1=$ não equivalente, $0=$ indeciso $\mathrm{e}+1=$ equivalente). Foi solicitado aos especialistas que, caso escolhessem as opções - 1 e 0 , colocassem suas sugestões no campo de observações. Após essa avaliação, as pesquisadoras reuniram as sugestões em um arquivo, programa Microsoft Word, e analisaram as sugestões dos especialistas. Já os dados quantitativos foram tabulados e analisados no programa Microsoft Excel 20, com o intuito de verificar a percentagem de concordância entre os especialistas, aplicandose a fórmula: divisão do número de concordantes especialistas pelo número total de especialistas $(n=7)$, e o resultado multiplicado por 100 . Para o presente estudo, foi estabelecido uma taxa de percentagem de concordância, entre os especialistas, mínima de $80 \%$, conforme indicação de algumas pesquisas (Lourenção \& Tronchin, 2016; Silva et al., 2016; Silva-Rocha \& Osório, 2017; Wynd et al., 2003). Após a análise dos dados, foi elaborada a Teacher Questionnaire Emotion - Versão brasileira 1.0 (TEQ - Versão brasileira 1.0).

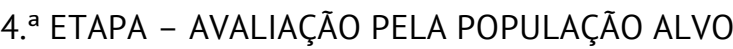

O Teacher Questionnaire Emotion - Versão brasileira 1.0 (TEQ - Versão brasileira 1.0) foi então submetida a avaliação pela população-alvo. Esta etapa tem o objetivo de verificar se o instrumento como um todo, i.e., instruções, itens e escala de resposta, é compreendido pela população-alvo (Borsa et al., 2012). Participaram, nesta etapa, três professoras da educação básica, com as características da população alvo. As professoras tinham formação na área de pedagogia $(n=2)$ e Educação Física $(n=1)$, especialização na área de neuropsicologia, modalidades de intervenção no processo de 
aprendizagem, ciência do movimento humano e Educação Física escolar, e tinham mais de oito anos de experiência profissional.

Nesse sentido, foi-lhes enviado, via e-mail, o questionário TEQ Versão brasileira 1.0 para a avaliação e foi-lhes solicitado para avaliarem a compreensão dos itens do TEQ - Versão brasileira 1.0. Para tal, tinham que ler cada item e em caso de não entendimento, escrever sugestões, podendo substituir palavras/sinônimos que fossem de mais fácil compreensão pela população alvo, i.e., professores (Borsa et al., 2012). Após essa etapa, foi criada a Teacher Questionnaire Emotion - Versão brasileira 1.1 (TEQ - Versão brasileira 1.1).

\section{5. ${ }^{a}$ ETAPA - TRADUÇÃO REVERSA}

Participou, nesta etapa, um tradutor profissional (bilíngue), nativo em língua inglesa e domínio da língua portuguesa, com mais de 21 anos de experiência em tradução. A tradução reversa consistiu em traduzir a TEQ - Versão brasileira 1.1 para o idioma inglês (escala original). 0 tradutor não tinha conhecimento da escala original e não tinha formação na área da psicologia. Dessa etapa, surgiu a versão retraduzida do TEQ (TEQ-RT) que foi comparada com a TEQ original.

\section{6. ${ }^{a}$ ETAPA - ESTUDO-PILOTO}

Para o estudo-piloto, foi selecionada uma amostra por conveniência, composta por 37 professores da educação básica (dos quais 94.6\% do sexo feminino) com idades compreendidas entre os 28 e 55 anos $(\mathrm{M}=39.89, \mathrm{DP}=$ 7.393). A maioria dos professores era formada em pedagogia (56.8\%) e Educação Física (35.1\%); três professoras referiram ainda possuir formação em letras $(n=2)$ e psicologia $(n=1)$. 0 tempo de atuação como professor variou entre 5 e 26 anos ou mais, sendo que a maioria dos professores (64.8\%) possuía mais que 11 anos de experiência profissional. A maioria dos professores tinha um curso de especialização (83.8\%).

Para a coleta de dados foram utilizados quatro instrumentos. Primeiramente, um questionário sociodemográfico, através do qual foram solicitadas algumas informações gerais, como idade, sexo, formação, curso de pós-graduação e tempo de atuação na docência. 0 segundo instrumento foi o TEQ - Versão brasileira 1.1. O terceiro instrumento foi o questionário de Avaliação da Praticabilidade de Instrumentos. Esse questionário é composto por três perguntas que têm como objetivo avaliar o tempo despendido e a facilidade no preenchimento de instrumentos de medida (e.g., TEQ), por meio de uma escala Likert de 5 pontos (discordo totalmente (1), discordo parcialmente (2), não tenho opinião (3), concordo parcialmente (4) e concordo totalmente (5) (Coluci \& Alexandre, 2009). Por fim, utilizou-se o instrumento de avaliação da compreensão verbal e da clareza dos itens, proposto por Canabarro (2018) e adaptado para este estudo, no que se refere aos itens avaliados. De acordo com este instrumento, os professores avaliaram a sua compreensão de cada item $(n=35)$ do TEQ - Versão brasileira 1.1, numa escala Likert de 6 pontos $(0=$ não entendi nada; $1=$ entendi pouco; $2=$ entendi razoavelmente; $3=$ entendi quase tudo, mas tenho dúvidas; $4=$ entendi quase tudo; $5=$ entendi por completo e não tenho dúvidas). Por último, foi solicitado para os professores escreverem qualquer dúvida ou dificuldade de entendimento referente a alguma palavra ou frase, sugerindo adequações. 
0 questionário, composto pelos quatro instrumentos, foi aplicado por meio eletrônico e os participantes assinaram (eletronicamente) o termo de consentimento livre e esclarecido, bem como foram informados sobre a natureza da pesquisa, benefícios previstos e eventuais constrangimentos em responder alguma questão do questionário. Se o participante se sentisse incomodado tinha direito de não responder, conforme as recomendações de pesquisas envolvendo seres humanos da Resolução nº 466/2012 do Ministério da Saúde/Conselho Nacional de Saúde (Brasil, 2012), Regulamento no 2016/679 do Parlamento Europeu e do Conselho da União Europeia, de 27 de abril de 2016 (Parlamento Europeu e Conselho da União Europeia, 2016) e Carta Ética do Instituto de Educação da Universidade de Lisboa (Deliberação n. ${ }^{\circ}$ 453/2016, do Conselho Científico). A análise de dados do estudo-piloto foi realizada no Statistical Package for the Social Sciences (SPSS), versão 24.0.

Após o estudo piloto, a versão TEQ-RT foi enviada para a apreciação da autora principal do TEQ original. Essa apreciação consistiu em verificar possíveis diferenças conceituais dos itens da escala original e da versão adaptada (Borsa et al., 2012), com o intuito de verificar discrepâncias entre a versão original e TEQ - Versão brasileira 1.1.

\section{RESULTADOS}

Os resultados são apresentados conforme as etapas do procedimento de adaptação cultural (Figura 1). Os dois tradutores buscaram realizar uma tradução adaptada para o contexto brasileiro do TEQ, entretanto, foram encontradas algumas diferenças nas traduções (23 itens). Essas diferenças se referiam a utilização de sinônimos ou mesmo na redação da frase, porém esses aspetos não alteravam o seu significado. Por exemplo, na afirmação "I am joyful when the class atmosphere is positive", o primeiro tradutor traduziu como "eu fico contente quando a atmosfera da aula é positiva", já o segundo tradutor traduziu como "fico alegre quando a atmosfera da aula é positiva". Após as sugestões do comitê de especialistas ficou como "eu fico alegre quando o clima da aula é positivo".

Na construção da versão síntese (TEQ-VCP) foram adaptados termos e, em alguns casos, utilizaram-se sinônimos para melhor compreensão dos professores. Além disso, as dúvidas que surgiram em relação a expressões idiomáticas do TEQ original, como nos itens "unfair distribution of work tasks gets me so frustrated that I get all sweaty" e "due to my students' achievements, I feel as if I am growing", foram compreendidas após contacto com a autora principal do questionário. Com isso foram realizadas as alterações necessárias para se alcançar equivalência conceitual dos itens.

Para a análise das equivalências da TEQ-VCP (tabela 1) foi estabelecido uma taxa de percentagem de concordância, entre os especialistas, mínima de $80 \%$, conforme indicação de alguns estudos (Lourenção \& Tronchin, 2016; Silva et al., 2016; Silva-Rocha \& Osório, 2017; Wynd et al., 2003). Dos 35 itens avaliados, 17 alcançaram a percentagem mínima nas equivalências: semântica, idiomática experiencial e conceitual (itens: $1,2,3,5,7,8,11,12,13,15,23,27,28,29,30,33$ e 35) (Tabela 1). Porém, 18 itens tiveram taxa menor que $80 \%$ em alguma das equivalências (itens, $4,6,16,17,18,19,20,21,22,24,32$ e 34), sendo que os itens $9,10,14$, 
25, 26 e 31 não atingiram essa percentagem mínima em nenhuma das equivalências e, como tal, foram revistos.

Para aumentar a equivalências dos itens que não alcançaram a percentagem mínima, foram realizadas algumas modificações indicadas pelos especialistas. As sugestões de adequações referiam-se a questões gramaticais, substituição de algum termo por sinônimos e acréscimo e/ou inversão de palavras na frase. No item 10, por exemplo, "eu me sinto feliz quando os alunos entendem o conteúdo" foi alterado para "eu fico feliz quando os alunos entendem o conteúdo da disciplina".

No geral, os especialistas sugeriram adequações para a maioria dos itens $(n=30)$. Essas sugestões foram analisadas, tendo-se em conta o contexto cultural brasileiro e o contexto escolar. Para a maioria dos itens foram introduzidas poucas alterações, por exemplo, no item 6 foi substituído "eu me sinto alegre..." por "eu fico alegre". Por fim, todas as sugestões foram acatadas criando-se a versão TEQ - Versão brasileira 1.0.

Tabela 1

Resultado da avaliação da equivalência pelos especialistas $(n=7)$

\begin{tabular}{|c|c|c|c|c|}
\hline \multirow{3}{*}{ Itens } & \multicolumn{4}{|c|}{ Equivalências } \\
\hline & Semântica & Idiomática & Experiencial & Conceitual \\
\hline & $\%$ & $\%$ & $\%$ & $\%$ \\
\hline Item 1 & 85,7 & 85,7 & 100,0 & 85,7 \\
\hline Item 2 & 100,0 & 100,0 & 100,0 & 100,0 \\
\hline Item 3 & 100,0 & 100,0 & 100,0 & 85,7 \\
\hline Item 4 & 71,4 & 100,0 & 100,0 & 100,0 \\
\hline Item 5 & 100,0 & 100,0 & 100,0 & 100,0 \\
\hline Item 6 & 71,4 & 85,7 & 85,7 & 85,7 \\
\hline Item 7 & 85,7 & 85,7 & 100,0 & 85,7 \\
\hline Item 8 & 100,0 & 100,0 & 100,0 & 100,0 \\
\hline Item 9 & 71,4 & 57,1 & 42,9 & 42,9 \\
\hline Item 10 & 57,1 & 57,1 & 71,4 & 57,1 \\
\hline Item 11 & 100,0 & 100,0 & 100,0 & 100,0 \\
\hline Item 12 & 85,7 & 100,0 & 85,7 & 85,7 \\
\hline Item 13 & 85,7 & 100,0 & 100,0 & 100,0 \\
\hline Item 14 & 57,1 & 71,4 & 71,4 & 71,4 \\
\hline Item 15 & 85,7 & 100,0 & 85,7 & 100,0 \\
\hline Item 16 & 71,4 & 57,1 & 85,7 & 85,7 \\
\hline Item 17 & 57,1 & 100,0 & 85,7 & 100,0 \\
\hline Item 18 & 71,4 & 100,0 & 100,0 & 100,0 \\
\hline Item 19 & 42,9 & 85,7 & 85,7 & 85,7 \\
\hline Item 20 & 85,7 & 71,4 & 100,0 & 85,7 \\
\hline Item 21 & 71,4 & 100,0 & 100,0 & 85,7 \\
\hline Item 22 & 71,4 & 85,7 & 85,7 & 100,0 \\
\hline Item 23 & 100,0 & 100,0 & 85,7 & 100,0 \\
\hline Item 24 & 57,1 & 85,7 & 71,4 & 85,7 \\
\hline Item 25 & 42,9 & 57,1 & 71,4 & 28,6 \\
\hline Item 26 & 71,4 & 71,4 & 71,4 & 71,4 \\
\hline Item 27 & 85,7 & 100,0 & 100,0 & 100,0 \\
\hline Item 28 & 85,7 & 100,0 & 100,0 & 100,0 \\
\hline Item 29 & 85,7 & 100,0 & 85,7 & 100,0 \\
\hline Item 30 & 85,7 & 85,7 & 100,0 & 100,0 \\
\hline Item 31 & 28,6 & 71,4 & 71,4 & 71,4 \\
\hline Item 32 & 71,4 & 85,7 & 85,7 & 85,7 \\
\hline Item 33 & 85,7 & 100,0 & 100,0 & 100,0 \\
\hline Item 34 & 71,4 & 85,7 & 71,4 & 85,7 \\
\hline Item 35 & 85,7 & 85,7 & 85,7 & 85,7 \\
\hline
\end{tabular}


$\mathrm{Na}$ avaliação pelo público-alvo, as professoras não tiveram dificuldade de compreensão dos itens. Entretanto, após sugestões, na busca de um melhor entendimento pelas professoras, optou-se por substituir dois termos. 0 termo "sobre-estimar" foi substituído pelo termo "superestimar", pelo fato de ser mais utilizado no cotidiano, bem como a palavra entorpecido(a) que foi substituída por desanimado(a). Com essas alterações surgiu a TEQ - Versão brasileira 1.1, que foi enviada para a realização da tradução reversa. A tradução reversa foi realizada de maneira satisfatória na comparação com a TEQ versão original.

No estudo-piloto, o tempo médio de preenchimento do TEQ Versão brasileira 1.1 foi de 6 minutos (DP = 1.841). Na tabela 2, encontram-se os resultados da avaliação da praticabilidade de instrumento pelos professores. Pode-se verificar que a maioria dos professores (86.5\%) concordou totalmente que as instruções do TEQ - Versão brasileira 1.1 são de fácil entendimento, bem como suas questões. A maioria (89.2\%) também concordou totalmente que é fácil assinalar as respostas do TEQ - Versão brasileira 1.1 .

Tabela 2

Avaliação da praticabilidade de instrumentos pelos professores $(n=37)$

\begin{tabular}{lccccc}
\hline Questões & $\begin{array}{c}\text { Discordo } \\
\text { Totalmente } \\
\mathrm{N}(\%)\end{array}$ & $\begin{array}{c}\text { Discordo } \\
\text { parcialmente } \\
\mathrm{N}(\%)\end{array}$ & $\begin{array}{c}\text { Não tenho } \\
\text { opinião } \\
\mathrm{N}(\%)\end{array}$ & $\begin{array}{c}\text { Concordo } \\
\text { parcialmente } \\
\mathrm{N}(\%)\end{array}$ & $\begin{array}{c}\text { Concordo } \\
\text { totalmente } \\
\mathrm{N}(\%)\end{array}$ \\
\hline $\begin{array}{l}\text { Eu achei fácil } \\
\text { entender as } \\
\text { instruções do } \\
\text { questionário }\end{array}$ & - & - & - & $3(8,1)$ & $34(91,9)$ \\
$\begin{array}{l}\text { Eu achei fácil } \\
\text { entender as } \\
\text { questões do } \\
\text { questionário }\end{array}$ & - & - & & & \\
$\begin{array}{l}\text { Eu achei fácil } \\
\text { assinalar as } \\
\text { respostas do } \\
\text { questionário. }\end{array}$ & - & & & & \\
\hline
\end{tabular}

No que diz respeito à compreensão e clareza de cada um dos 35 itens do TEQ - Versão brasileira 1.1, verificou-se que a maioria dos itens $(\mathrm{n}=30)$ foram compreendidos pelos professores $(98.3 \%$ entenderam por completo e não tiveram dúvidas e $1.3 \%$ entenderam quase tudo). Apenas 0.4 $\%$ dos professores afirmaram que nos itens 3, 5, 8, 9 e 22 "entenderam quase tudo, mas tiveram dúvidas". Estes resultados quantitativos corroboram alguns relatos dos professores sobre a compreensão dos itens do TEQ Versão brasileira 1.1, por exemplo, "não identifiquei dificuldade em responder e necessidade de consultar palavras desconhecidas, pois fazem parte do nosso cotidiano" (professora, 49 anos).

Por fim, a autora principal do TEQ original propôs adequações em alguns termos do TEQ - Versão brasileira 1.1. Após a realização dos mesmos a autora aprovou a versão final, denominada de Teacher Questionnaire Emotion (TEQ) - Versão brasileira (TEQ - Versão brasileira). 


\section{DISCUSSÃO}

O processo de adaptação cultural de instrumentos tem como intuito buscar equivalência entre o instrumento original e o de destino (Beaton et al., 2000). Para tal, não existe um consenso na literatura sobre um método padrão para ser utilizado, todavia, não é simplesmente traduzir o instrumento original para o idioma de destino. Tendo em conta a importância do contexto cultural na expressão e compreensão de emoções, há necessidade da utilização de um rigoroso procedimento metodológico para se alcançar um instrumento de medição confiável (Borsa et al., 2012; Sousa \& Rojjanasrirat, 2011).

A literatura sugere algumas etapas e, apesar de não obedecer a mesma ordem, tem em comum as seguintes fases: autorização do(s) autor(es) da escala original, tradução, tradução reversa, comitê de especialistas e pré-teste/estudo-piloto (Beaton et al., 2000; Borsa et al., 2012; ITC, 2017; Sousa \& Rojjanasrirat, 2011; WHO, 2020).

O objetivo desse estudo foi traduzir o TEQ para a língua portuguesa e adaptá-lo para a cultura brasileira. Para alcançar esse propósito, seguiu-se etapas propostas na literatura citadas anteriormente (Beaton et al., 2000; Borsa et al., 2012; WHO, 2020). Também se aplicou algumas recomendações, tais como, tradutores bilíngues, comissão de avaliadores: 6-10 especialistas, estudo-piloto: 30-40 sujeitos, avaliação do autor(es) do instrumento original (Beaton et al., 2000; Sousa \& Rojjanasrirat, 2011).

0 processo de tradução foi realizado de maneira satisfatória pelos dois tradutores. Dessa etapa, surgiram duas versões distintas e, posteriormente, analisadas. Os tradutores possuem vasta experiência em tradução, bem como, são formados em áreas distintas, o que contribuiu para uma tradução adequada na maioria dos itens, a nível conceitual e da terminologia cotidiana. Beaton et al. (2000) alertam que a boa qualidade da tradução passa pela escolha de tradutores qualificados. Na etapa síntese, procurou-se construir uma única versão, atendendo-se às equivalências semântica, idiomática, experiencial e conceitual, pois é fundamental garantir que a tradução tenha equivalência com o instrumento original e, simultaneamente, que seja compreensível pela população-alvo (Borsa et al., 2012).

A avaliação do comitê de especialistas foi de fundamental importância, visto que suas sugestões foram pertinentes e as pesquisadoras aceitaram todas as sugestões, tendo sido realizadas adequações na maioria dos itens, o que permitiu uma maior clareza na linguagem e equivalência semântica, idiomática, experiencial e conceitual (Beaton et al., 2000). Essa etapa contribuiu para a construção do instrumento adaptado com melhor compreensão pelo público-alvo, o que pode ser confirmado na análise das três professoras ( $4^{\circ}$ etapa) e posteriormente no estudo-piloto.

Os resultados do estudo-piloto mostraram que o TEQ - Versão brasileira 1.1 tem elevada concordância de compreensão, nas instruções, no geral e é de fácil preenchimento. Na análise individual de cada item, de uma maneira geral os professores não tiveram dificuldade de entendimento, o que revela boa compreensão dos termos e conceitos utilizados. 0 estudo-piloto é uma etapa importante do processo de adaptação cultural, visto que é por meio dele que se pode avaliar se o instrumento está preparado para ser utilizado, quer dizer, se possui boa compreensão pelo público a que se destina (Borsa et al., 2012), no caso do presente estudo, os professores. 
A tradução reversa revelou que o original e a versão brasileira do TEQ são equivalentes a nível conceitual, conforme aprovação da autora principal do TEQ original. A tradução reversa é um cuidado a mais no processo de tradução, visto que por meio dela é possível identificar possíveis discrepâncias conceituais entre os itens do instrumento original e os itens do instrumento adaptado (Beaton et al., 2000). A similaridade entre a versão da tradução reversa e o instrumento original pode revelar uma aceitável adaptação (ITC, 2017). Por último, a versão final foi aprovada pela autora principal da escala original.

É de referir que, sendo a adaptação cultural um importante passo para a validação cultural de um instrumento, a conclusão da sua validação requer ainda a realização de estudos de avaliação das propriedades psicométricas (Borsa et al., 2012). Portanto, o (TEQ) - Versão brasileira está adaptado para a cultura brasileira e pronto para a avaliação das propriedades psicométricas.

Em conclusão, estudar as emoções dos professores é de fundamental relevância, não só pelas demandas profissionais que a atuação docente exige e que podem afetar o bem-estar emocional do professor (Bahia et al., 2013; Fried et al., 2015), mas também por ter impactos no processo ensino-aprendizagem e consequentemente afetar a sua qualidade (Chen, 2016). 0 presente estudo de adaptação cultural do TEQ realizou o primeiro passo para disponibilizar um instrumento confiável e válido sobre as emoções dos professores, com o intuito de preencher lacunas ainda existentes, no que se refere ao estudo das emoções dos professores no contexto cultural brasileiro com método quantitativo. 0 próximo passo, que já está a ser realizado, é a exploração das propriedades psicométricas e o estudo de validade do TEQ - Versão brasileira.

\section{AGRADECIMENTOS}

Este artigo foi realizado no âmbito de uma investigação de Doutoramento em Educação, financiada pela Fundação para a Ciência e a Tecnologia - FCT (SFRH/BD/145367/2019).

As autoras agradecem aos tradutores, especialistas e professores que tornaram essa pesquisa possível. Agradecem ainda a Doutora Irena Burić, autora principal do TEQ, pela colaboração no processo de adaptação.

\section{REFERÊNCIAS}

Bahia, S., Freire, I., Amaral, A., \& Estrela, M. A. (2013). The emotional dimension of teaching in a group of Portuguese teachers. Teachers and Teaching: Theory and Practice, 19(3), 275-292. https://doi.org/10.1080/13540602.2012.754160

Beaton, D. E., Bombardier, C., Guillemin, F., \& Ferraz, M. B. (2000). Guidelines for the process of crosscultural adaptation of self-report measures. Spine, 25(24), 3186-3191. https://doi.org/10.1097/00007632-200012150-00014

Borsa, J. C., Damásio, B. F., \& Bandeira, D. R. (2012). Adaptação e validação de instrumentos psicológicos entre culturas: Algumas considerações. Paidéia, 22(53), 423-432. https://doi.org/10.1590/S0103-863X2012000300014

Brasil. Ministério da Saúde. Conselho Nacional de Saúde. (2012). Resolução n. 466, de 12 de dezembro de 2012. Aprova diretrizes e normas regulamentadoras de pesquisas envolvendo seres humanos. Brasília, Diário Oficial 
da União, 12 dez. 2012.

https://bvsms.saude.gov.br/bvs/saudelegis/cns/2013/res0466_12_12_2012.html

Burić, I., Slišković, A., \& Macuka, I. (2018). A mixed-method approach to assessment of teachers' emotions: Development and validation of teacher emotion questionnaire (TEQ). Educational psychology, 38(3), 325-349.

https://doi.org/10.1080/01443410.2017.1382682

Canabarro, R. C. C. (2018). Consultoria colaborativa: Influências na autoeficácia docente e no processo de inclusão de alunos com transtorno do espectro autista [Doctoral thesis, Universidade Federal de Santa Maria, Santa Maria, Brasil]. Repositório Digital da UFSM. https://repositorio.ufsm.br/bitstream/handle/1/16256/TES_PPGEDUCACAO_2018_CA NABARRO_RENATA.pdf?sequence $=1$ \&isAllowed $=y$

Chen, J. (2016). Understanding teacher emotions: The development of a teacher emotion inventory. Teaching and Teacher Education, 55, 68-77. https://doi.org/10.1016/j.tate.2016.01.001

Chen, J. (2019). Exploring the impact of teacher emotions on their approaches to teaching: A structural equation modeling approach. British Journal of Educational Psychology, 89, 57-74. https://doi.org/10.1111/bjep.12220

Coluci, M. Z. O., \& Alexandre, N. M. C. (2009). Development of a questionnaire to evaluate the usability of assessment instruments. Revista Enfermagem UERJ, 17(3), p. 378-382.

Deliberação n. ${ }^{\circ}$ 453/2016, de 15 de março de 2016, Conselho Científico do Instituto de Educação da Universidade de Lisboa, Diário da República, 52, 2. ${ }^{\text {a }}$ série, 9153-9154.

Freire, I., Bahia, S., Estrela, M., \& Amaral, A. (2014). A dimensão emocional da docência: Contributo para a formação de professores. Revista Portuguesa de Pedagogia, 46(2), 151-171. https://doi.org/10.14195/1647-8614_46-2_8.

Frenzel, A. C., T. Goetz, T., Stephens E. J., \& Jacob, B. (2009). Antecedents and effects of teachers' emotional experiences: An integrated perspective and empirical test. In P. A. Schutz \& M. Zembylas (Eds.), Advances in teacher emotion research: The impact on teachers' lives (pp. 129-151). Springer.

Frenzel, A. C., Pekrun, R., Goetz, T., Daniels, L. M., Durksen, T. L., BeckerKurz, B., \& Klassen, R. M. (2016). Measuring teachers' enjoyment, anger, and anxiety: He teacher emotions scales (TES). Contemporary Educational Psychology, 46, 148-163. https://doi.org/10.1016/j.cedpsych.2016.05.003

Fried, L., Mansfield, C., \& Dobozy, E. (2015). Teacher emotion research: Introducing a conceptual model to guide future research. Issues in Educational Research, 25(4), 415-441.

Hargreaves, A. (2000). Mixed emotions: Teachers' perceptions of their interactions with students. Teaching and Teacher Education, 16, 811-826.

https://doi.org/10.1016/S0742-051X(00)00028-7

Hong, J., Heddy, B., Ruan, J., You, S., Kambara, H., Nie, Y., \& Monobe, G. (2016). Revising and validating achievement emotions questionnaire - teachers (AEQ-T). International Journal of Educational Psychology, 5(1), 80-107.

https://doi.org/10.17583/ijep.2016.1395

International Test Commission (2017). The ITC Guidelines for translating and adapting tests (2nd ed.).

https://www.intestcom.org/files/guideline_test_adaptation_2ed.pdf

Lourenção, D. C. A., \& Tronchin, D. M. R. (2016). Segurança do paciente no ambiente cirúrgico: Tradução e adaptação cultural de instrumento validado. Acta Paulista de Enfermagem, 29(1), 1-8. https://doi.org/10.1590/1982-0194201600002 
Parlamento Europeu e Conselho da União Europeia (2016). Regulamento (UE) 2016/679 do Parlamento Europeu e do Conselho de 27 de abril de 2016 relativo à proteção das pessoas singulares no que diz respeito ao tratamento de dados pessoais e à livre circulação desses dados e que revoga a Diretiva 95/46/CE (Regulamento Geral sobre a Proteção de Dados). https://op.europa.eu/pt/publication-detail//publication/3e485e15-11bd-11e6-ba9a-01aa75ed71a1/language-pt

Scherer, K. R. (2005). What are emotions? And how can they be measured? Social Science Information, 44(4), 695-729.

https://10.1177/0539018405058216

Schutz, P. A., Hong, J. Y., Cross, D. I., \& Osbon, J. N. (2006). Reflections on investigating emotion in educational activity settings. Educational Psychology Review, 18(4), 343-360. https://doi.org/10.1007/s10648-006-9030-3

Sheppard, M., \& Levy, S. A. (2019). Emotions and teacher decisionmaking: An analysis of social studies teachers' perspectives. Teaching and Teacher Education, 77, 193-203. https://doi.org/10.1016/j.tate.2018.09.010

Silva, M. C., Peduzzi, M., Sangaleti, C. T., Silva, D., Agreli, H. F., West, M. A., $\&$ et al. (2016). Cross-cultural adaptation and validation of the teamwork climate scale. Revista de Saúde Pública, 50(52), 1-10. http://doi.org/10.1590/S15188787.2016050006484

Silva-Rocha, V. V., \& Osório, F. L. (2017). Cross-cultural adaptation of the Sport Anxiety Scale-2 (SAS-2) for the Brazilian context. Trends in Psychiatry and Psychotherapy, 39(3), 202-206. https://doi.org/10.1590/2237-6089-2017-0027

Slišković, A., Burić, I., \& Sorić, I. (2019). The relations between principal support and work engagement and burnout: Testing the tole of teachers' emotions and educational level. Work, 64(2), 203- 215. https://doi.org/10.3233/WOR-192987

Sousa, V. D., \& Rojjanasrirat, W. (2011), Translation, adaptation and validation of instruments or scales for use in cross-cultural health care research: A clear and user-friendly guideline. Journal of Evaluation in Clinical Practice, 17, 268274. https://doi.org/10.1111/j.1365-2753.2010.01434.x

Sutton, R. E., \& Wheatley, K. F. (2003). Teachers' emotions and teaching: A review of the literature and directions for future research. Educational Psychology Review, 15(4), 327-358. https://doi.org/10.1023/A:1026131715856

Taxer, J. L., \& Frenzel A. C. (2015). Facets of teachers' emotional lives: A quantitative investigation of teachers' genuine, faked, and hidden emotions. Teaching and Teacher Education, 49, 78-88. https://doi.org/10.1016/j.tate.2015.03.003

$\mathrm{Xu}, \mathrm{Y}$. (2018). A methodological review of $\mathrm{L} 2$ teacher emotion research: Advances, challenges and future directions. In J. de. D. Martinez Agudo (Ed.), Emotions in second language teaching: Theory, research and teacher education (pp. 35-49). Springer. https://doi.org/10.1007/978-3-319-75438-3_3

World Health Organization [WHO] (2020, May 10). Process of translation and adaptation of instruments.

https://www.who.int/substance_abuse/research_tools/translation/en/

Wynd, C. A., Schmidt, B., \& Schaefer, M. A. (2003). Two quantitative approaches for estimating content validity. Western Journal of Nursing Research, 25(5), 508-518. https://doi.org/10.1177/0193945903252998

Zembylas, M. (2007). Emotional ecology: the intersection of emotional knowledge and pedagogical content knowledge in teaching. Teaching and Teacher Education, 23, 355-367. https://doi.org/10.1016/j.tate.2006.12.002

Zembylas, M., \& Schutz, P. A. (2009). Research on teachers' emotions in education: Findings, practical implications and future agenda. In P. A. Schutz \& M. 
Zembylas (Eds.), Advances in teacher emotion research: the impact on teachers' lives (pp. 367-377). Springer. https://doi.org/10.1007/978-1-4419-0564-2_18

Informação dos autores:

i Universidade de Lisboa, Instituto de Educação, Portugal. https://orcid.org/0000-0002-8511-1601

ii Universidade de Lisboa, Instituto de Educação, Portugal. https: //orcid.org/0000-0002-9394-9738

Toda a correspondência relativa a este artigo deve ser enviada para:

Camila Marta de Almeida

Rua Diogo Afonso, Lote 2, 2580-474 Carregado, Alenquer, Portugal.

camila.almeidaecampus.ul.pt

Recebido em 14 de agosto de 2020

Aceite para publicação em 21 de outubro de 2020 


\title{
Cultural adaptation of an instrument to measure teachers' emotions (TEQ)
}

\begin{abstract}
The study of teachers' emotions has been gaining prominence, and emotions are currently recognized as an essential dimension affecting teachers' work. Despite having gained relevance in the educational area, there is a need for valid instruments that measure teachers' discrete emotions. This study aimed to translate the Teacher Emotion Questionnaire (TEQ) to the Portuguese language and to adapt it to the Brazilian culture. Fifty-one participants were involved: four translators, seven specialists, 40 teachers, and also the researchers and the main author of the original TEQ. The current methodological study followed six stages: (1) translations; (2) synthesis of the translated versions; (3) committee of experts; (4) evaluation by the target audience; (5) reverse translation; (6) pilot study. The translation and synthesis steps were carried out properly, and some changes were made after the assessment by the experts and target audience. The pilot study showed that the adapted instrument is easy to understand and fill in. Finally, after some adjustments, the cultural adaptation was approved by the main author of the original TEQ. This study was the first step to provide a reliable and valid instrument on the emotions of teachers. The next step will be the exploration of the psychometric properties of TEQ - Brazilian version.
\end{abstract}

Keywords: Teachers' emotions; Cultural adaptation;

Teacher Emotion Questionnaire 


\section{Adaptación cultural de un instrumento para evaluar las emociones del profesor (TEQ)}

\section{RESUMEN}

El estudio de las emociones de los profesores ha ganado destaque, y actualmente es reconocido como una dimensión esencial del trabajo docente. Sin embargo, a pesar de haber ganado relevancia en el área educativa, es visible que hay una laguna de instrumentos cuantitativos válidos para medir las emociones discretas del profesor. Este estudio tuvo como objetivo traducir al portugués y adaptar el Teacher Emotion Questionnaire (TEQ) para la cultura brasileña. Participaron 51 individuos, de ellos cuatro traductores, siete especialistas, 40 profesores, y también las investigadoras y autora principal del TEQ original. El presente estudio metodológico de adaptación cultural contó con las etapas a continuación: (1) traducciones; (2) síntesis de las versiones traducidas; (3) comité de expertos; (4) evaluación por el público objetivo; (5) traducción inversa; (6) estudio piloto. Las etapas de traducción y síntesis fueron realizadas adecuadamente, introduciendo algunas modificaciones después del análisis de los especialistas y del público objetivo. El estudio piloto mostró que el instrumento adaptado es de fácil comprensión y llenado. Finalmente, la adaptación cultural fue aprobada por la autora principal del TEQ original, después de algunos ajustes. El presente estudio dio el primer paso para colocar a disposición un instrumento confiable y válido para analizar las emociones de los profesores. $\mathrm{El}$ próximo paso será la exploración de las propiedades psicométricas del TEQ versión brasileña.

Palabras-clave: Emociones del profesor; Adaptación cultural; Teacher Emotion Questionnaire 